\title{
Role of Vitamins in Advanced Therapy for Parkinson's Disease: Decoding the Paradox
}

Keywords: Vitamins, Peripheral neuropathy, LCIG

doi:10.1017/cjn.2021.106

Can J Neurol Sci. 2022; 49: 3-4

The relationship between vitamins and Parkinson's disease (PD) has been explored in many clinical studies, animal models, and single-cell experiments. In PD, vitamins act as cofactors in enzymatic pathways of dopamine metabolism, affect pharmacokinetics of levodopa, and play neuroprotective roles by mitigating neuroinflammation and oxidative stress.

A critical role of central and peripheral inflammation has been emphasized in the recent literature regarding the onset and progression of PD. ${ }^{1-3}$ Supplementation of vitamins with known anti-inflammatory, antioxidant, and free radicle scavenging properties is thus one of the major neuroprotective strategies under recent focus in PD. ${ }^{4,5} \mathrm{~A}$ recent Swedish trial has demonstrated an inverse correlation between dietary vitamins $\mathrm{E}$ and $\mathrm{C}$ intake and the risk of $\mathrm{PD}$, especially in overweight and obese patients. ${ }^{6}$ Vitamin $\mathrm{C}$ can enhance levodopa absorption that can help to manage 'delayed on' or 'poor on' response in PD patients. Ascorbic acid also plays a major role in the biosynthesis of norepinephrine in sympathetic neurons and thus is a potential therapeutic target for managing orthostatic hypotension in PD. ${ }^{7}$ Some studies have shown a correlation of vitamin D receptor polymorphism with risk of PD, though the results are inconsistent. $^{8}$ Similarly, two prospective trials have shown contradictory results on the relationship between mid-life serum vitamin D levels and the risk of PD. ${ }^{9,10}$ Vitamin D supplementation has also been noted to improve balance and decrease fall risk in PD patients. However, the dose- and time-dependent effects of these vitamins need to be evaluated further. ${ }^{11,12}$

Apart from vitamins E, C, and D, vitamin B complex also has a major impact in PD management. In the current issue of the Journal, Taher et al. have highlighted the physiology and pathophysiology of vitamins $\mathrm{B}_{6}$ (pyridoxine), $\mathrm{B}_{9}$ (folate), and $\mathrm{B}_{12}$ (cobalamin) in PD patients undergoing levodopa-carbidopa intestinal gel (LCIG) therapy. ${ }^{13}$ An anonymous survey conducted in February 2020 among neurologists, gastroenterologists, and clinical nurses from across Canada has served as a prelude to the manuscript. The survey clearly revealed the knowledge gap and inconsistencies among individual practices on multiple facets in this regard. Thus, the unmet need of a therapeutic recommendation had to be addressed.

Peripheral neuropathy is a well-known complication of chronic levodopa therapy. Studies have shown that cumulative doses of levodopa, deficiencies of vitamins $\mathrm{B}_{6}, \mathrm{~B}_{9}, \mathrm{~B}_{12}$, and genetic factors play major roles in causing neuropathy and result in elevation of methylmalonic acid (MMA) and homocysteine. Neuropathic complications can be acute like Guillain-Barré syndrome, subacute, and chronic. ${ }^{14}$ Patients receiving levodopa daily dose (LDD) of beyond 2,000 $\mathrm{mg}$ and in whom LDD is rapidly increased within a short interval, like during the initiation of LCIG therapy, are at high risk of developing this side effect. ${ }^{15}$ High homocysteine and low pyridoxine are the major culprits on this regard. Two pathophysiological mechanisms have been highlighted: (1) Co-administration of levodopa and dopa decarboxylase inhibitor (carbidopa) increases levodopa metabolism by catechol-O-methyltransferase (COMT) that converts it to 3-O-methyldopa. For this conversion, COMT requires S-adenosylmethionine (SAM) as the methyl-group donor and that in turn facilitates conversion of SAM into S-adenosylhomocysteine and subsequently increased homocysteine production. Homocysteine can enter either into the transmethylation cycle and convert into methionine by methionine synthase (vitamin $\mathrm{B}_{12}$ and folate as cofactors) or into transsulfuration pathway and converts into cystathionine by cystathionine beta-synthase (vitamin $\mathrm{B}_{6}$ as co-factor). In case of deficiency of these vitamins, neurotoxic effects of hyperhomocysteinemia manifest; and (2) Carbidopa irreversibly binds to pyridoxal phosphate (PLP, active form of vitamin $\mathrm{B}_{6}$ ) and permanently deactivates PLP-dependent enzymes like many decarboxylases and depletes pyridoxine reserve pool. ${ }^{13}$ While vitamin $\mathrm{B}_{6}$ deficiency can cause small fiber neuropathy, epilepsy, and encephalopathy, vitamin $\mathrm{B}_{12}$ deficiency can lead to large fiber neuropathy or dorsal column involvement. Autonomic neuropathy involving visceral nerves can cause gastroparesis that can lead to constipation and insufficient absorption of levodopa and dietary vitamins, further enhancing the vicious cycle.

The authors have also discussed two potential mechanisms explaining why the risk of vitamin deficiencies and peripheral neuropathy is more common during LCIG therapy in comparison to oral levodopa: (i) methylcellulose gel used in LCIG may hinder absorption of the dietary vitamins through jejunal membrane; and (ii) continuous delivery of LCIG may saturate the one-carbon pathway being used in levodopa metabolism because of the absence of 'metabolic rest' needed to utilize those vitamins for other metabolic functions. ${ }^{13}$

Finally, the paper has nicely summarized a therapeutic guideline on testing and managing vitamin deficiencies in PD patients undergoing LCIG therapy. Vitamin $\mathrm{B}_{12}$, homocysteine, and MMA must be tested before starting LCIG, after 6 months, and then once a year. Nuances of these tests should be kept in mind

Received ApriL 29, 2021. Final Revisions Submitted May 3, 2021. Date of Acceptance May 5, 2021. 
while ordering them to avoid misinterpretation of laboratory test results. Vitamin $\mathrm{B}_{6}$ and/or folate should be measured in scenarios of raised homocysteine with normal MMA and/or total vitamin $\mathrm{B}_{12}$. Plasma PLP, serum/red blood cell folate, and total $\mathrm{B}_{12}$ and/or MMA are recommended tests for vitamin $\mathrm{B}_{6}, \mathrm{~B}_{9}$, and $\mathrm{B}_{12}$. Nerve conduction study should be done before starting LCIG to look for any pre-existing neuropathy and once a year subsequently to monitor the development or progression of neuropathy. Prophylactic vitamin $\mathrm{B}_{12}$ and vitamin $\mathrm{B}_{6}$ supplementation is needed for high-risk individuals. However, clinicians must be aware of the neurotoxic side effects of excess vitamin $\mathrm{B}_{6}$ and potential risk of inducing neuropathy with folate supplementation in patients with vitamin $\mathrm{B}_{12}$ deficiency. COMT inhibitors can be added to manage hyperhomocysteinemia. Therapeutic dose of cyanocobalamin, folic acid, and pyridoxine is needed to manage vitamin deficiencies and resulting side effects like peripheral neuropathy. In advanced cases, temporary discontinuation of LCIG therapy may be needed along with supportive therapeutic alternatives like subcutaneous apomorphine.

Clinicians are using advanced therapeutic options like LCIG to manage motor fluctuation more efficiently and to offer a better quality of life in PD patients. However, the complex pharmacology and biochemistry underneath it should be understood clearly to avoid potential side effects. Adequate knowledge of monitoring and treating those side effects is also important. Having said that, the clear dose-response relationship between the vitamins and their therapeutic effect has not been established. Especially in scenarios like PD, where brain biochemistry and body physiology are already altered, fine-tuning the supply of micronutrients to match with the changing metabolic demands is much harder than it seems. Further prospective studies are needed to address these issues.

\section{Disclosures}

Dr. Ganguly reports grant from Academic Medical Organization of Southwestern Ontario (AMOSO), outside the submitted work. Dr. Tuesta Bernaola reports grant from Parkinson society of Southwestern Ontario (PSSO), outside the submitted work. Dr. Jog reports grants from Parkinson society Canada, grants from CIHR, grants from NSERC, grants from MITACS, grants from Research Council of Norway, grants from Parkinson Society of Southwestern Ontario, grants from Dystonia Medical Research Foundation, other from Allergan, other from Abbvie, other from Merz Pharmaceuticals, other from Boston Scientific, other from Valeo Pharma, other from Sunovion, other from Paladin Labs, grants from Academic Medical Organization of Southwestern Ontario (AMOSO), outside the submitted work.

\section{Statement of Authorship}

JG and MTB wrote the first draft of the manuscript; critically reviewed by $\mathrm{MJ}$.

Jacky Ganguly

London Movement Disorder Centre, London Health Sciences Centre, University of Western Ontario, London, Ontario, Canada
Mellany Tuesta Bernaola

London Movement Disorder Centre, London Health Sciences Centre, University of Western Ontario, London, Ontario, Canada

Mandar Jog

London Movement Disorder Centre, London Health Sciences Centre, University of Western Ontario, London, Ontario, Canada

Correspondence to: Mandar Jog, London Movement Disorder Centre, London Health Sciences Centre, University of Western Ontario, 339 Windermere Road, London, Ontario N6A 5A5, Canada. Email: mandar.jog@lhsc.on.ca

\section{REFERENCES}

1. Metta V, Leta V, Mrudula KR, et al. Gastrointestinal dysfunction in Parkinson's disease: molecular pathology and implications of gut microbiome, probiotics, and fecal microbiota transplantation. J Neurol. 2021. doi: 10.1007/s00415-021-10567-w.

2. Pajares M, Rojo AI, Manda G, Boscá L, Cuadrado A. Inflammation in Parkinson's disease: mechanisms and therapeutic implications. Cells. 2020;9:1-32.

3. Herrick MK, Tansey MG. Is LRRK2 the missing link between inflammatory bowel disease and Parkinson's disease? NPJ Park Dis. 2021;7:26.

4. Zhao X, Zhang M, Li C, Jiang X, Su Y, Zhang Y. Benefits of vitamins in the treatment of Parkinson's disease. Oxid Med Cell Longev. 2019;2019:1-14.

5. Zhang SM, Hernan MA, Chen H, Spiegelman D, Willett WC, Ascherio A. Intakes of vitamins $\mathrm{E}$ and $\mathrm{C}$, carotenoids, vitamin supplements, and PD risk. Neurology. 2002;59:1161-9.

6. Hantikainen E, Trolle Lagerros Y, Ye W, et al. Dietary antioxidants and the risk of Parkinson disease: the Swedish national march cohort. Neurology. 2021;96:e895-903.

7. Benarroch EE. What is the role of ascorbic acid in norepinephrine synthesis and orthostatic hypotension? Neurology. 2020;95: 913-6.

8. Wang X, Shen N, Lu Y, Tan K. Vitamin D receptor polymorphisms and the susceptibility of Parkinson's disease. Neurosci Lett. 2019;699:206-11.

9. Knekt P, Kilkkinen A, Rissanen H, Marniemi J, Sääksjärvi K, Heliövaara M. Serum vitamin D and the risk of Parkinson disease. Arch Neurol. 2010;67:808-11.

10. Shrestha S, Lutsey PL, Alonso A, Huang X, Mosley TH, Chen H. Serum 25-hydroxyvitamin D concentrations in Mid-adulthood and Parkinson's disease risk. Mov Disord. 2016;31:972-8.

11. Man Anh H, Linh DM, My Dung V, Thi Phuong Thao D. Evaluating dose- and time-dependent effects of vitamin $\mathrm{C}$ treatment on a Parkinson's disease fly model. Parkinsons Dis. 2019;2019:1-14.

12. Fullard ME, Duda JE. A review of the relationship between vitamin D and Parkinson disease symptoms. Front Neurol. 2020;11:1-11.

13. Taher J, Naranian T, Poon Y-Y, et al. Vitamins and infusion of levodopa-carbidopa intestinal gel. Can J Neurol Sci. 2021;49:19-28. doi: 10.1017/cjn.2021.78.

14. Müller T, Van Laar T, Cornblath DR, et al. Peripheral neuropathy in Parkinson's disease: Levodopa exposure and implications for duodenal delivery. Parkinsonism Relat Disord. 2013;19:501-7.

15. Loens S, Chorbadzhieva E, Kleimann A, Dressler D, Schrader C. Effects of levodopa/carbidopa intestinal gel versus oral levodopa/ carbidopa on B vitamin levels and neuropathy. Brain Behav. 2017;7:e0698. 